\title{
Los futuros maestros ante el problema de la contaminación de los mares por plásticos y el consumo
}

\author{
Mercedes Jaén \\ Departamento de Didáctica de las Ciencias Experimentales. Universidad de Murcia. España. \\ mjaen@um.es \\ ORCID: http:// orcid.org/0000-0001-5935-4979 \\ Patricia Esteve \\ Departamento de Didáctica de las Ciencias Experimentales. Universidad de Murcia. España. \\ p.esteve@um.es \\ ORCID: bttp:/ / orcid.org/0000-0003-4768-1969

\section{Isabel Banos-González} \\ Departamento de Didáctica de las Ciencias Experimentales. Universidad de Murcia. España. \\ ibbg1@um.es \\ ORCID: https:/ / orcid.org/0000-0002-2569-1210
}

[Recibido: 18 Enero 2018. Revisado: 10 Julio 2018. Aceptado: 4 Agosto 2018]

\begin{abstract}
Resumen: En este trabajo se aborda desde el ámbito educativo el problema de la contaminación de los mares por residuos. En un escenario en el que la contaminación por plásticos se ha convertido en un problema de orden global, indagamos sobre las ideas, actitudes y competencias didácticas que muestran los futuros maestros cuando se enfrentan a los hábitos de consumo de nuestra sociedad actual. Se analiza la puesta en práctica de una intervención educativa en la que, a partir de una situación problemática vinculada a la contaminación por plásticos, los estudiantes realizan tareas en las que exploran el problema y algunos de sus hábitos de consumo. Sus respuestas indican que establecen relaciones causales entre ciertas actividades humanas y las problemáticas ambientales, y se muestran dispuestos a asumir compromisos para reducir sus consumos, aunque lo hacen desde un escenario limitado a su entorno y sus intereses. Al implementar esta problemática en Primaria, la mayoría utilizan un modelo simple basado en la información. Se han identificado algunas dificultades al concretar estrategias que permitan desarrollar el pensamiento crítico en los niños y niñas de Primaria.
\end{abstract}

Palabras clave: Sostenibilidad; Futuros maestros; Contaminación plásticos; Consumo; Propuestas.

Future teachers facing the problem of pollution of the seas by plastics and consumption

Abstract: In this work, the problem of pollution of the seas by waste is addressed from the educational field. In a context in which contamination by plastics has become a problem of global order, we inquire about the ideas, attitudes and didactic competences that future teachers show when they facing the consumption habits of our current society. The implementation of an educational intervention is analysed, in which, from a problematic situation related to pollution by plastics, students perform different tasks. By means of these tasks, they explore the problem and some of their consumption habits. Their responses indicate that they establish causal relationships between certain human activities and environmental problems, and are willing to assume commitments to reduce their consumption, although they do so from a scenario limited to their surrounding and their interests. When implementing this problem in Primary school, most of them use a simple model based on information. Some difficulties have been identified in specifying strategies that allow the development of critical thinking in Primary school pupils.

Keywords: Sustainability; Future teachers; Pollution by plastics; Consumption; Proposals.

Para citar este artículo: Jaén, M., Esteve, P., Banos-González, I. (2019) Los futuros maestros ante el problema de la contaminación de los mares por plásticos y el consumo. Revista Eureka sobre Enseñanza y Divulgación de las Ciencias 16(1), 1501. doi: 10.25267/Rev_Eureka_ensen_divulg_cienc.2019.v16.11.1501 


\section{La cultura de lo efímero: el plástico, un habitual en nuestras vidas}

El exceso de consumo de bienes y servicios en los países industrializados constituye uno de los conflictos socioambientales más importantes en la actualidad, que además, ha potenciado un desequilibrio entre nuestra población, el medio ambiente y otros países (Blaser, Feit y McRae 2004).

La armonía con el entorno ha quedado difuminada ante la perspectiva de mejorar la calidad de vida y el bienestar de los ciudadanos, desde un enfoque exclusivamente económico. Sin embargo, indicadores que van más allá de este enfoque, constatan que la calidad de vida ha descendido en paralelo a la degradación de los sistemas naturales, lo que confirma que ambas esferas -sociedad y medio ambiente- tienen una interacción dinámica e interdependiente (Happiness Planet 2012). Por tanto, el incremento del bienestar no es el responsable directo de la degradación ambiental, sino su concepción mercantilista, ligada a un estilo de vida consumista y contaminante.

Sin embargo, de forma continuada, se generan nuevas necesidades que promueven un consumo incesante. El consumismo se ha instaurado como una seña de identidad de nuestra sociedad y, aunque es axiomático en la vida de niños y jóvenes, éstos no consideran que compren de modo innecesario (Gámez 2010).

Entre los grandes problemas ligados al consumismo está la producción masiva de residuos y su llegada a los sistemas naturales. Un claro ejemplo es la contaminación de los mares y océanos por basuras, que se ha convertido en un problema de magnitud planetaria en poco más de medio siglo. Se estima una entrada anual al mar de 6,4 millones de toneladas de basura, unos 200 kilos cada segundo, donde el plástico es el principal componente y su procedencia deriva, en un 80\%, de actividades terrestres (UNEP 2009).

En España, los resultados del Programa del Seguimiento de Basuras Marinas en Playas muestran que por cada 100 metros de costa, se detectan una media de 377 residuos, situándose entre los más comunes los fragmentos de plástico inferiores a $2,5 \mathrm{~cm}$, botellas de plástico y envoltorios (Ministerio de Agricultura, Pesca, Alimentación y Medio Ambiente 2017).

El ritmo de producción de plásticos va en aumento y se estima que se llegará a 500 millones de toneladas en 2020 (Greenpeace 2016). La mayor parte se emplean en la fabricación de productos de un único uso, lo que refleja la futilidad con la que se utiliza este material y el desafío que supone en el marco de nuestra economía, la reducción de su uso. Además, según datos nacionales, la mitad de los plásticos que entran a sistemas de gestión no se reciclan y terminan en vertederos. Respecto a los que se abandonan, las cifras alcanzan unos 30 millones de envases, fundamentalmente latas y botellas (Plastics Europe 2017).

Las consecuencias son 5,25 trillones de plásticos flotantes que forman grandes vórtices de basura, acentuados por los grandes giros de circulación del agua superficial marina. Aunque se encuentran en todos los océanos, el de mayor dimensión está localizado en el Pacífico Norte y ocupa entre los 1,7 y 3,4 millones de $\mathrm{km}^{2}$.

Los efectos de esta contaminación son especialmente alarmantes. Además de la asfixia, heridas, malformaciones, etc., que sobre todo afectan a cetáceos y aves, los microplásticos podrían estar pasando a la cadena trófica marina; lo que además de problemas físicos, implica la incorporación de tóxicos a la biomasa marina (Eriksen, Barendziak, Haskamp y Kastenholz 2014).

El turismo también se ve considerablemente afectado, en especial en economías dependientes de este sector. La UE invierte en la limpieza de costas y playas unos 630 millones de euros al año (Comisión Europea 2018). Sin embargo, muchas sociedades costeras de subsistencia, 
pertenecientes a países de menor desarrollo económico y muy dependientes de la pesca y el turismo, difícilmente pueden enfrentar estos gastos, aunque son las más perjudicadas, a pesar de no ser las responsables del nivel de contaminación actual.

\section{Acciones educativas para afrontar las problemáticas ambientales en la formación de profesores}

El plástico es un material que ha pasado a ser parte fundamental de nuestra vida, pero quizá sus repercusiones no son bien comprendidas por la sociedad. Desde el ámbito educativo, los problemas generados por su consumo, no nos deben dejar indiferentes, entre otras razones, porque los educadores debemos implicarnos en tareas de concienciación ciudadana que permitan afrontar las posibles amenazas para la vida en el planeta.

Educar en un consumo responsable es necesario en el camino hacia una sociedad más equitativa y comprometida, para lo cual, se debería promover de forma tenaz una ética del consumo basada más en la libertad y la solidaridad, que en la capacidad y necesidad de consumir (Cortina 2002).

El planteamiento educativo del conflicto generado por el consumo y producción de residuos plásticos, nos puede permitir plantear a los futuros maestros dos aspectos:

- Superar la percepción general de que la degradación ambiental se debe, básicamente, a grandes industrias contaminadoras (Gallup Organization 2010), y reconozcan que los hábitos individuales pueden tener efectos acumulativos que suponen cambios globales.

- Afrontar la responsabilidad individual desde un proceso de reflexión sobre la necesidad de cambios en nuestros comportamientos (Grace y Byrne 2010).

Para que nuestros estudiantes puedan hacer frente a los desafíos de la sostenibilidad de forma creativa y crítica, será necesario favorecer una adecuada percepción de los graves problemas socioambientales a los que nos enfrentamos y capacitarlos para tomar decisiones dirigidas a lograr un futuro sostenible $y$, de forma prioritaria, incorporarlo como competencia en la formación de los docentes (Vilches y Gil 2013). Además, tendría un efecto multiplicador, puesto que los docentes en formación serán los responsables de la enseñanza de las nuevas generaciones durante 30 años.

No obstante, la identificación de las competencias clave en sostenibilidad que deben poseer los estudiantes postgraduados ha sido objeto de un amplio debate en el ámbito académico. En la revisión crítica realizada por Wiek, Withycombe y Redman (2011) se sintetizan las contribuciones más relevantes y en un contexto de investigación en sostenibilidad y resolución de problemas, y se concluye con la definición e integración de las competencias clave en sostenibilidad: competencia de pensamiento sistémico, competencia anticipatoria, competencia normativa, competencia estratégica y competencia interpersonal. El interés de este estudio está, sobre todo, en su utilización para el diseño y evaluación de programas relacionados con la sostenibilidad. En este sentido, Vega-Marcote, Varela-Losada y Álvarez-Suárez (2015) evalúan el desarrollo de estas competencias en un programa educativo, dirigido a futuros maestros, en el que participan individual y colectivamente en la solución de problemas socioambientales. Los resultados en las competencias de sostenibilidad les permiten concluir la eficacia del modelo para favorecer el desarrollo de acciones sostenibles.

Otro aspecto a considerar son las representaciones sociales que se utilizan para interpretar el mundo y actuar ante conflictos de intereses que nos afectan de modo directo. En la construcción de ese escenario intervienen distintos procesos cognitivos relacionados con: conocimientos adquiridos en el contexto educativo, la experiencia personal, medios de 
comunicación, procesos de interacción social, etc., que dotan de coherencia nuestras actitudes y valores (Meira 2013).

En la disposición para adoptar determinadas acciones para mitigar los efectos de los problemas ambientales influyen factores como las preocupaciones personales, sociales y éticas, muy dependientes del contexto. Un objetivo fundamental como profesores será incrementar la relevancia social de estos temas, abriendo espacios para la discusión, poniendo énfasis en que los estudiantes adquieran un sentido crítico ante estos conflictos socioambientales, y asuman responsabilidades enfrentándolos a sus conductas de consumo (Collins- Figueroa 2012).

Respecto a los planteamientos metodológicos, hemos de ser conscientes que los estudiantes no cambian sus ideas automáticamente al recibir nueva información más ajustada a la verdad científica. Al abordar estos conflictos en las aulas, debemos evitar realizar propuestas que describan los fenómenos de forma aislada e independiente de las actividades humanas (Vilches y Gil 2013). Estos enfoques dificultan la comprensión de los problemas complejos y su vinculación al entorno desde un enfoque sistémico, en el cual el medioambiente se considera un sistema constituido por factores físicos y socio-culturales interrelacionados entre sí, entre los que se producen acciones, reacciones y ajustes, de tal forma que evoluciona con el tiempo (Aramburu 2000).

Aunque la escuela es un espacio adecuado para promover el consumo responsable frente a los impactos ambientales debidos a nuestros hábitos, por diferentes causas, no siempre desarrolla este papel. Los maestros no se sienten suficientemente formados para llevar a la práctica estas temáticas en sus aulas desde orientaciones innovadoras, a pesar de que consideran que la conservación de nuestro entorno es un contenido relevante en Primaria (Lindemann-Matthies, Constantinou, Lehnert, Nagel, Raper y Kadji 2011). Con frecuencia, los docentes se centran en la adquisición de conocimientos sobre los fenómenos naturales, haciendo hincapié en las importantes relaciones que se producen en el denominado "medio natural", sin tener en cuenta las actividades humanas, ni los conflictos derivados de ellas, y consideran estar realizando actividades en el ámbito de la Educación para la Sostenibilidad, cuando en realidad, los objetivos de aprendizaje se centran más en el ámbito disciplinar de la Biología (López Rodríguez y Jiménez Aleixandre 2001).

Si queremos promover compromisos para un futuro sostenible, en las propuestas dirigidas a la formación de maestros, hemos de destacar la importancia de analizar críticamente las amenazas que subyacen en la relación entre los seres humanos y los problemas ambientales, y reflexionar sobre la necesidad de que los ciudadanos participen en la conservación ambiental (Gayford 2000). En este sentido, la toma de decisiones participativa e inclusiva es una estrategia muy eficaz para formar a los maestros que pueden suponer el punto de partida necesario para involucrarse activamente en la sostenibilidad y hacer partícipes a sus alumnos (Sims y Falkenberg 2013).

Según Cebrián y Junyent (2014), es preciso promover, desde la formación inicial de profesores, la adquisición de competencias sobre estrategias educativas, mediante las cuales identificar responsabilidades sobre los problemas, las causas y sus efectos, y puedan participar en las medidas necesarias para su control o solución. Este propósito implica una integración de la formación disciplinar y didáctica para lograr, por un lado, la adquisición de conocimientos, valores y actitudes; y por otro, desarrollar competencias para diseñar propuestas de enseñanza dirigidas a promover la sostenibilidad desde la escuela (Elster, Barendziak, Haskamp y Kastenholz 2014).

Sobre el desarrollo de estas competencias didácticas, Esteve (2016) analiza la implementación de una propuesta basada en el planteamiento de distintos problemas socioambientales. En los 
resultados se reconoce que los futuros maestros progresan desde enfoques de enseñanza centrados en los conceptos, a otros más dirigidos a promover actitudes y comportamientos. En sus valoraciones, los profesores en formación consideran fundamental que se ofrezcan oportunidades para reflexionar sobre la conservación del medio ambiente y aceptan que se trata de contenidos esenciales para la etapa de Primaria. Son capaces de identificar como relevante que los escolares adopten compromisos y participen activamente. Sin embargo, asumen que no se sienten preparados para abordar planteamientos innovadores orientados a promover actitudes y comportamientos pro-ambientales en el aula.

En coherencia con lo anterior, y teniendo presente nuestros objetivos en la formación del profesorado, hemos diseñado y evaluado una acción educativa dirigida a implicar a los futuros maestros en un problema ambiental relevante y lograr cambios efectivos en sus ideas y compromisos. En concreto, se pretenden alcanzar los siguientes objetivos específicos:

- Indagar sobre sus dificultades para identificar las causas y los efectos acumulativos de algunas actividades humanas cotidianas que generan residuos que pueden producir impactos sobre la biodiversidad marina y las personas.

- Valorar su capacidad para asumir compromisos y responsabilidades que conduzcan a proponer cambios realistas en sus hábitos que les permita reducir su consumo de plásticos.

- Analizar desde las orientaciones de la Educación para la Sostenibilidad, la competencia didáctica que muestran al diseñar una salida a la playa para abordar la problemática de contaminación marina en la escuela.

\section{Metodología}

\section{Contexto de nuestro estudio}

Este estudio se desarrolla en el marco del Grado de Primaria de la Universidad de Murcia, en la asignatura de $2^{\circ}$ curso Enseñanza y Aprendizaje del Medio Natural I. En la investigación participan 60 futuros maestros organizados en 15 grupos de trabajo.

Para abordar los objetivos establecidos, se ha planificado una intervención educativa de cuatro horas, basada en el planteamiento de una situación problemática, cuyo eje temático es la existencia del gran vórtice de basura del océano Pacífico Norte.

\section{Descripción de la intervención didáctica}

La propuesta se plantea mediante un problema cuyo enunciado propone un hipotético crucero a la Isla Basura del océano Pacífico, constituida por residuos plásticos en distintos grados de densidad. Mediante su desarrollo, los futuros profesores han de indagar sobre la influencia del ser humano y algunas de las consecuencias de nuestros hábitos de consumo sobre situaciones concretas de degradación ambiental.

Al plantear el problema, los estudiantes utilizan sus ideas iniciales para identificar las causas de la formación de ese vórtice y la responsabilidad que pudieran tener sobre su existencia, mediante estas cuestiones: ¿Conocías la existencia de esta isla? ¿Cuáles son las causas del problema que ha generado esta isla? ¿'Te consideras responsable de la Isla Basura? ¿Por qué?

Posteriormente, se presenta la siguiente información sobre la contaminación por plásticos en los océanos y otros lugares de planeta:

- Características del vórtice y datos sobre producción mundial de plásticos.

- Periodo de degradación de distintos tipos de plásticos.

- Datos sobre la cantidad de residuos obtenidos en las labores de limpieza de costas al año. 
- Listados de tipos de objetos encontrados en el mar.

- Contenido intestinal de peces comerciales.

- Noticias reales y ejemplos sobre cómo afectan los plásticos a diferentes especies de animales, a la pesca y a la salud de las personas.

En primer lugar, los futuros profesores analizan la documentación centrándose en los dos primeros documentos, es decir, en la formación de la Isla Basura, sus causas y su potencial contribución al problema. Mediante la respuesta a las cuestiones de la Hoja de trabajo (ANEXO) han de reflexionar también sobre la cantidad y el tipo de residuos plásticos que producen a lo largo de un año y su peligrosidad para la vida marina.

Una vez examinados todos los documentos, deberán vincular sus hábitos de consumo con la generación de residuos y su impacto en los ecosistemas marinos. La respuesta a las cuestiones de la Hoja de informe final (ANEXO) permite que los futuros profesores analicen las principales consecuencias de la contaminación por plásticos, su nivel de responsabilidad en la generación del problema y proponer soluciones viables.

Como tarea final, para valorar su competencia didáctica en relación a la enseñanza de estos contenidos, los futuros profesores han de diseñar una salida a la playa en la que participarían los niños y niñas de tercer ciclo de Primaria con sus familias. Este tipo de actividad se ha propuesto debido al interés que muestran los futuros profesores por la realización de salidas al entorno. Se plantea mediante una noticia de prensa sobre la limpieza de playas por parte de voluntarios y se indica que deben diferenciar tres fases en su diseño. En los objetivos se remarcó que, además de realizar tareas de limpieza, debería promoverse la concienciación y participación activa ante la problemática de contaminación marina.

La información sobre sus ideas iniciales procede de las respuestas durante el planteamiento del problema. Mientras que los conocimientos que manifiestan ante los efectos de la contaminación por plásticos, sus hábitos y sus soluciones, se ha obtenido mediante el análisis de la Hoja de trabajo (HT) y la Hoja de informe final (HIF), que realizaron de forma individual, aunque trabajaban en grupo, durante el desarrollo del problema. La competencia didáctica se ha analizado mediante la valoración de las actividades que proponen para realizar en la playa.

\section{Criterios de análisis de los resultados}

Las respuestas se han analizado mediante la identificación de categorías inductivas-deductivas y sus frecuencias. Se han diferenciado cuatro dimensiones:

- Las ideas iniciales sobre el origen del vórtice, sus posibles causas y responsabilidades ante la generación del problema ambiental.

- Las causas y consecuencias que identifican en la contaminación por plásticos y los tipos de impactos que consideran: ambientales, sociales y económicos. De este modo, podemos determinar cómo interpretan las circunstancias del conflicto y establecer posibles dificultades en este sentido.

- Sus comportamientos personales y la asunción de responsabilidades, se valoran en función del compromiso que manifiestan. Igualmente, al analizar sus medidas de solución, se diferencia entre propuestas que no exceden del ámbito personal y estrategias que pretenden involucrar activamente a la sociedad (Lindemann-Matthies 2002).

- Los planteamientos metodológicos de las actividades de enseñanza que proponen para Primaria y su potencialidad para concienciar a sus futuros estudiantes y a sus familias sobre la contaminación ligada a los plásticos y la necesidad de actuar, se han valorado mediante diez criterios adaptados de las orientaciones metodológicas propuestas desde la Educación para la 
Sostenibilidad por diferentes autores (CESIRE-CDEC 2008, Gámez 2010, Morgensen, Mayer, Breiting y Varga 2009, Tilbury 2011) (Tabla 1). Como indicadores se ha considerado el cumplimiento o no de dichos criterios.

Tabla 1. Criterios utilizados en el análisis de las actividades propuestas por los futuros profesores.

1. La actividad parte de buenas preguntas más que de explicaciones sobre la contaminación de los mares y océanos por plásticos.

2. Da oportunidad a los alumnos de expresar y confrontar sus propias ideas sobre la problemática.

3. Está contextualizada en situaciones próximas a los estudiantes.

4. Promueve el interés, la autonomía y la iniciativa, implicándolos en su desarrollo.

5. Propicia un aprendizaje interactivo entre los alumnos sobre el problema de contaminación marina por plásticos y promueve la discusión en el aula.

6. Permite incidir en las causas de la contaminación y su relación con actividades humanas habituales.

7. Muestra los efectos de nuestro consumismo en la conservación de la biodiversidad marina y/o el bienestar de otras personas.

8. Facilita el pensamiento crítico de los alumnos respecto a sus comportamientos como consumidor.

9. Invita a asumir compromisos guiando a los niños y niñas a comprender su papel para conservar la biodiversidad marina.

10. Fomenta la transferencia de los aprendizajes logrados hacia ámbitos familiares o en el entorno próximo.

\section{Resultados}

Describiremos los resultados a partir de las respuestas obtenidas en las tareas desarrolladas durante nuestra intervención didáctica. Así, expondremos en primer lugar las ideas de los futuros docentes al plantearles el problema y, posteriormente, analizaremos sus proposiciones durante y al final de la actividad, contrastando con las ideas iniciales.

\section{Ideas iniciales de los futuros maestros sobre el problema}

Al enfrentarse al problema, los futuros docentes muestran un desconocimiento casi completo sobre la existencia del vórtice y, en general, sobre cuestiones de contaminación de los mares y océanos. Sólo de forma puntual, aluden a noticias sobre islas deshabitadas llenas de residuos o situaciones similares que han conocido mediante los medios de comunicación. Al establecer las causas, solo siete estudiantes las asocian con actividades humanas relacionadas con el consumo, la producción de residuos y la ausencia de reciclaje (Tabla 2). No obstante, todos consideran muy importante difundir la existencia de esta zona para mostrar el efecto de la contaminación debida a los residuos plásticos.

Tabla 2. Causas del problema de contaminación por plásticos

\begin{tabular}{|l|c|}
\hline Causas del problema & Frecuencias \\
\hline No las conoce & 36 \\
\hline Sólo se refiere al enunciado & 5 \\
\hline Otros escenarios similares de contaminación & 7 \\
\hline Actividades de los seres humanos y elevada producción de residuos & 5 \\
\hline
\end{tabular}

Sobre las responsabilidades que admiten, hemos agrupado sus respuestas según el nivel de compromiso que manifiestan. En el primer grupo responden en primera persona, es decir, plantean una responsabilidad a nivel individual, mientras que el resto aluden a obligaciones genéricas, empleando fórmulas como el impersonal o la tercera persona. En las razones que esgrimen no se observan diferencias, en ambos casos se refieren a un exceso de consumo y a una enorme producción de residuos (Tabla 3). 
Tabla 3. Responsabilidades sobre el problema y razones.

\begin{tabular}{|c|c|c|}
\hline \multicolumn{2}{|l|}{ Responsabilidades } & Frecuencias \\
\hline \multirow{2}{*}{ Responsabilidad individual } & Exceso de consumo y compras & 5 \\
\hline & Producción masiva de residuos & 9 \\
\hline \multirow{4}{*}{ Responsabilidad colectiva } & Porque hay un exceso de consumo en la sociedad & 2 \\
\hline & Producción masiva de residuos en la sociedad & 23 \\
\hline & Respuestas genéricas & 10 \\
\hline & No conocen el problema & 11 \\
\hline
\end{tabular}

Sus respuestas aluden a la elevada cantidad de basura que generamos en el día a día, los vertidos de residuos en lugares inadecuados como el váter o el fregadero, o la falta de reciclaje. Por otra parte, reconocen que consumen productos plásticos sin considerar los posibles impactos que producen. Algunos señalan que deberíamos tener en cuenta los tipos de envases o embalajes de los productos que compramos.

Otro grupo de estudiantes considera que el desconocimiento de este problema no les permite valorar sus responsabilidades, incluido en el grupo que no se siente comprometido individualmente. Como respuestas genéricas hemos agrupado los planteamientos poco rigurosos o indefinidos, como «no estoy concienciada», «todos somos responsables» o «no cuidamos el medio ambiente».

\section{Resultados sobre las causas del problema y las consecuencias producidas sobre el medio marino por la contaminación por plásticos}

Después de analizar la información suministrada, al analizar las causas que han generado esta "isla de basura" (Item $2 \mathrm{HT}$ ), los futuros profesores, excepto cuatro, señalan diversas actividades humanas como responsables, lo cual determina una gran diferencia respecto a sus ideas iniciales (Tabla 2). Sin embargo, se centran en un origen inmediato del problema, es decir en vertidos directos de plásticos al mar. Prácticamente todos señalan las actividades recreativas en playas y barcos, como las principales responsables de dicha acumulación. De forma complementaria, algunos mencionan las basuras de origen urbano que pudieran ser arrastradas mediante el viento. En ningún caso argumentan sobre la variable tiempo en el proceso de formación o su origen indiscutiblemente continental. Sólo de modo excepcional se refieren al consumo excesivo de plásticos por parte de los ciudadanos o al escaso reciclaje.

No obstante, cuando valoran la importancia de que la sociedad conozca la existencia de estas zonas (Ítem $1 \mathrm{HT}$ ), consideran a partes iguales que podría favorecer una mayor concienciación sobre la magnitud del problema y una mayor implicación ciudadana en su reducción.

$\mathrm{Al}$ valorar la abundancia y peligrosidad en los mares de los residuos plásticos al mismo tiempo que analizan la cantidad que ellos producen (Ítem 3, 4, 5 HT), las respuestas de los futuros profesores evidencian una relación entre su consumo y peligrosidad a nivel general (Tabla 4).

Tabla 4. Relación entre los productos que utilizan y su peligrosidad y abundancia

\begin{tabular}{|c|c|c|c|}
\hline Tipo de residuo plástico & $\begin{array}{c}\text { Consumo medio anual } \\
\text { (cantidad) }\end{array}$ & $\begin{array}{c}\text { Residuos más } \\
\text { abundantes }\end{array}$ & $\begin{array}{c}\text { Residuos más } \\
\text { peligrosos }\end{array}$ \\
\hline Botellas de agua & 344 & ++ & ++ \\
\hline Bolsas & 623 & ++ & - \\
\hline Envoltorios & 650 & ++ & - \\
\hline Briks & 400 & ++ & ++ \\
\hline
\end{tabular}


Señalan principalmente las botellas de plástico y las bolsas como los residuos más abundantes y dañinos. El hecho de que sean productos que consumen con frecuencia, por ejemplo, una media de 623 bolsas/año, podría haber facilitado la asociación de la problemática con acciones de consumo cotidianas. Cuando plantean medidas para reducir estos consumos (Ítem 6 HT), la mayoría proponen fórmulas como reutilizar los botellines o usar cantimploras, bolsas de tela o fiambreras. Solo cinco estudiantes manifiestan que no son capaces de reducir su consumo porque no saben cómo hacerlo o porque piensan que deben ser las empresas y establecimientos los que lo hagan.

$\mathrm{Al}$ calcular el consumo anual que realizan de estos productos, todos destacan que es superior a lo que pensaban, en especial el de las bolsas de plástico, que algunos alumnos reconocen usar más de 3000/año.

Cuando se pide a los estudiantes que valoren las consecuencias de esta contaminación por plásticos (Ítem $1 \mathrm{HIF}$ ), todos mencionan cómo afecta a los animales marinos, las fotos de animales atrapados en plástico es lo que ha resultado más impactante para ellos. No obstante, algunos también señalan impactos sociales o económicos (Tabla 5).

Los impactos de tipo social los enfocan hacia el riesgo que supone para la salud la presencia de plástico en variedades de peces comerciales o a los peligros de ingerir las toxinas derivadas de este material. En menor medida señalan impactos de tipo económico referidos a la disminución de la producción pesquera o de peor calidad.

Tabla 5. Tipos de impacto que consideran como consecuencias del problema

\begin{tabular}{|l|c|l|}
\hline Tipo de impacto & Frecuencias & Ejemplo de respuesta \\
\hline Ambiental & 23 & $\begin{array}{l}\text { «Algunos animales confunden las basuras con comida, como los delfines que se comen las } \\
\text { bolsas de plástico, o las aves,.....se mueren muchos animales por esto.» }\end{array}$ \\
\hline Ambiental y social & 23 & $\begin{array}{l}\text { «Este problema nos afecta a varios niveles, porque si el plástico entra en la cadena trófica, } \\
\text { al final, además de dañar a los animales marinos, también puede suponer problemas de } \\
\text { salud.» }\end{array}$ \\
\hline $\begin{array}{l}\text { Ambiental } \\
\text { económico }\end{array}$ & 2 & $\begin{array}{l}\text { «El mar está tan contaminado que las especies mueren, porque se asfixian o se } \\
\text { atragantan y los ecosistemas desaparecen, y entonces también la pesca. Se pierden negocios } \\
\text { pesqueros.» }\end{array}$ \\
\hline $\begin{array}{l}\text { Ambiental, socio- } \\
\text { económico }\end{array}$ & 12 & $\begin{array}{l}\text { «Las consecuencias son muchas, porque si hay plásticos en el mar los animales pueden } \\
\text { morir y esto, al final, influye en la pesca, hay menos y con los riesgos de que no sea } \\
\text { saludable, porque si los plásticos liberan toxinas no es sano.» }\end{array}$ \\
\hline
\end{tabular}

Estos resultados sugieren que solo establecen relaciones sencillas y evidentes entre los elementos y factores que intervienen en la generación de las problemáticas ambientales. Reconocen efectos directos desde la perspectiva de una sociedad desarrollada con buen nivel de vida. Así, se muestran preocupados por cómo puede afectar a su salud y a ciertos intereses económicos relacionados con la pesca, olvidando las consecuencias de tipo global, así como la cuestión de la sobreexplotación pesquera.

\section{Resultados sobre los compromisos, responsabilidades que asumen y propuestas de cambio}

En este apartado ha resultado muy interesante analizar la evolución de las ideas de los futuros profesores, ya que al ser conscientes de sus propios consumos, han de proponer cambios para solucionar el problema. Aunque se asume que el hecho de realizar buenas propuestas no necesariamente implica que los estudiantes vayan a cambiar sus hábitos, sí lo consideramos un paso significativo en el proceso de reconocer su capacidad de intervención como ciudadanos. 
Al intentar que concreten sobre si los residuos que producen podrían formar parte de esa acumulación de plásticos (Ítem $2 \mathrm{HIF}$ ), todos salvo seis, responden que sí. No obstante, el proceso mediante el cual han podido llegar allí no lo definen adecuadamente, mayoritariamente insisten en la acción de los vientos y las corrientes o aluden a errores de gestión de los vertederos que permiten que la lluvia arrastre estos productos hacia el mar.

Cuando han de reflexionar sobre lo que han aprendido (Ítem 3 HIF) destacan la magnitud de la contaminación por plásticos en los mares y los daños que provoca sobre la biodiversidad y las personas. También reconocen su concienciación sobre la importancia de la gestión de residuos -incluido el reciclaje-, e involucrar a sus familias para que también lo hagan. Sin embargo, al referirse a posibles cambios en sus hábitos, no ponen el acento en la reducción del consumo.

Respecto a su nivel de responsabilidad sobre el problema (Ítem 4 HIF), se observa un ligero avance de sus respuestas hacia posiciones más comprometidas, aunque continúan posicionándose más como responsables a nivel colectivo que individual. Solo tres futuros profesores continúan manteniendo que ellos no son responsables del problema (Tabla 6).

Tabla 6. Ideas finales sobre las responsabilidades

\begin{tabular}{|l|c|}
\hline Responsabilidades & Frecuencias \\
\hline $\begin{array}{l}\text { No, porque yo no tiro basura al mar, es responsabilidad de los barcos y la gente que deja } \\
\text { restos en la playa. }\end{array}$ & 3 \\
\hline Sí, me considero responsable como ciudadano y procuro no utilizar bolsas de plástico & 18 \\
\hline Sólo en parte, porque si esos residuos los recogieran del mar no existiría este problema. & 2 \\
\hline Sí, el estilo de consumo de nuestra sociedad es el responsable. & 37 \\
\hline
\end{tabular}

$\mathrm{Al}$ proponer soluciones viables frente a este problema (Ítem $5 \mathrm{HIF}$ ) de forma mayoritaria tienden a combinar distintos tipos de propuestas, aunque principalmente se refieren a la necesidad de concienciar a los ciudadanos, la reducción de su propio consumo y la imposición de normativas (Tabla 7).

Tabla 7. Soluciones propuestas para resolver el problema

\begin{tabular}{|l|c|}
\hline Soluciones viables & No $^{\text {estudiantes }}$ \\
\hline La concienciación de la sociedad & 40 \\
\hline Una reducción de mi consumo & 37 \\
\hline La imposición de normativas & 20 \\
\hline Colaboración activa con asociaciones o proyectos & 13 \\
\hline Reciclaje & 9 \\
\hline
\end{tabular}

Consideran de enorme importancia fomentar compromisos sostenibles desde la infancia haciendo a los escolares partícipes de estas problemáticas. Se refieren de modo general al conocimiento que los ciudadanos deberían tener sobre estos escenarios de contaminación, incidiendo en la colaboración de los medios de comunicación para difundir estas situaciones. Sin embargo, la reducción del consumo, lo plantean a título personal, justificando la necesidad de comenzar desde cambios individuales. En general, sus respuestas muestran una progresión respecto a sus razonamientos iniciales, y son más los que reconocen la importancia de disminuir sus consumos.

De forma complementaria, algunos consideran necesario la implantación de normativas u otras formas de control para evitar la contaminación por basuras o para promover el reciclaje. 
Solo algunos mencionan la relevancia de las asociaciones y de los grupos de acción social que pueden colaborar en limpiezas de playas o campañas de divulgación.

\section{Resultados de sus propuestas para abordar la problemática en Primaria}

La tarea de diseñar una salida a una playa dirigida a los niños y niñas de Primaria junto a sus familias, la abordaron en grupo, por lo que en total se diseñaron 15 propuestas. En ellas diferenciaban tres fases: previa, durante y después de la salida.

En la fase previa, utilizando distintos recursos (imágenes, historias, vídeos), proponen aportar información sobre el problema guiándolos para que reconozcan los aspectos clave.

Durante la salida a la playa, básicamente proponen la recogida y separación de residuos. En la mayoría de las actividades, se incluía el registro de éstos, de modo que se pudiera estudiar su composición o sus posibles orígenes. En ocasiones, proponen la toma de imágenes para, después, realizar murales, incluirlas en redacciones o para una puesta en común final.

No obstante, algunos grupos plantean organizar la jornada como una competición centrada en los residuos recogidos, lo cual podría desviar la atención sobre el origen de esa basura. Parecen confiar en que los escolares serían capaces de establecer relaciones entre las informaciones de la fase previa sobre la contaminación y el juego de la playa.

En la fase final, todos incorporan un análisis sobre los resultados obtenidos en la salida y una reflexión sobre las posibilidades de intervenir en el problema. En la mayoría de sus propuestas, invitan a asumir compromisos individuales en relación a los cambios de hábitos necesarios para reducir la generación de residuos. Estos son similares a los propuestos por ellos; sólo cinco grupos promueven compromisos colectivos para llevar a cabo un seguimiento posterior de la playa, realizar exposiciones, charlas, cartas dirigidas a un diario local o contactar con otros centros para implicarlos en el mantenimiento del litoral. Incluso, de modo excepcional, sugieren unirse a asociaciones o campañas para la conservación de los mares.

Al aplicar los criterios de análisis relacionados con la Educación para la Sostenibilidad, se aprecia que un poco más de la mitad de las propuestas cumplen seis o más de los diez posibles, aunque ninguna consigue contemplarlos todos. En general, aunque los resultados indican el cumplimiento de gran número de estos indicadores, la distribución es muy heterogénea y se detectan omisiones que inciden directamente en su efectividad para generar competencias en sostenibilidad (Tabla 8).

Al profundizar en los resultados observamos que en todos los casos propician aprendizajes interactivos en los que las niñas y los niños expresan y confrontan sus ideas mediante discusiones de aula. En contraposición, solo tres propuestas fomentan la transferencia de los aprendizajes, a pesar de que la actividad se debe organizar junto a las familias y tienen la posibilidad de plantear acciones conjuntas.

Dentro de la diversidad de las propuestas, los criterios que se cumplen mayoritariamente están relacionados con un papel "activo" de los alumnos, dotándolos de cierta autonomía, dando oportunidades para que confronten sus ideas, centrándose en las consecuencias ambientales del problema e invitándolos a asumir compromisos. En cambio, cumplen de forma más minoritaria los criterios relacionados con el desarrollo de la actividad, resaltando el papel de la información sobre el problema. Mención aparte merece que el desarrollo del pensamiento crítico, esencial en nuestra intervención, solo se cumple en ocho casos.

Desde un análisis global de estos resultados, solamente un tercio cumple al menos ocho de los diez criterios. Estos resultados no son muy alentadores, pues de poner en práctica sus propuestas, muy pocas actividades estarán contextualizadas en la vida diaria, será difícil la concienciación sobre los propios consumos y resultará complejo tratar de reducirlos. Por otra 
parte, dado el escaso papel de las familias en las distintas fases de la salida -la mayoría de las tareas las realizarían los escolares- será difícil la transferencia de los aprendizajes.

Tabla 8. Análisis de las propuestas atendiendo a los criterios de sostenibilidad

\begin{tabular}{|c|c|c|c|c|c|c|c|c|c|c|c|c|c|c|c|c|}
\hline \multirow{2}{*}{ Criterios } & \multicolumn{10}{|c|}{ PROPUESTAS } & \multirow{2}{*}{ CUMPLEN } \\
\hline & 1 & 2 & 3 & 4 & 5 & 6 & 7 & 8 & 9 & 10 & 11 & 12 & 13 & 14 & 15 & \\
\hline 1 & $\checkmark$ & - & - & - & $\checkmark$ & $\checkmark$ & $\checkmark$ & - & - & - & $\checkmark$ & $\checkmark$ & - & - & $\checkmark$ & 7 \\
\hline 2 & $\checkmark$ & - & $\checkmark$ & - & $\checkmark$ & $\checkmark$ & $\checkmark$ & - & $\checkmark$ & - & $\checkmark$ & $\checkmark$ & - & $\checkmark$ & $\checkmark$ & 10 \\
\hline 3 & $\checkmark$ & - & - & - & - & $\checkmark$ & $\checkmark$ & - & - & - & $\checkmark$ & $\checkmark$ & - & - & - & 5 \\
\hline 4 & $\checkmark$ & - & - & $\checkmark$ & $\checkmark$ & $\checkmark$ & $\checkmark$ & $\checkmark$ & - & $\checkmark$ & $\checkmark$ & - & $\checkmark$ & - & $\checkmark$ & 10 \\
\hline 5 & $\checkmark$ & $\checkmark$ & $\checkmark$ & $\checkmark$ & $\checkmark$ & $\checkmark$ & $\checkmark$ & $\checkmark$ & $\checkmark$ & $\checkmark$ & $\checkmark$ & $\checkmark$ & $\checkmark$ & $\checkmark$ & $\checkmark$ & 15 \\
\hline 6 & $\checkmark$ & - & - & - & - & $\checkmark$ & $\checkmark$ & - & - & - & $\checkmark$ & - & $\checkmark$ & - & $\checkmark$ & 6 \\
\hline 7 & $\checkmark$ & $\checkmark$ & $\checkmark$ & $\checkmark$ & $\checkmark$ & $\checkmark$ & - & $\checkmark$ & - & $\checkmark$ & $\checkmark$ & $\checkmark$ & $\checkmark$ & $\checkmark$ & $\checkmark$ & 13 \\
\hline 8 & - & - & $\checkmark$ & - & $\checkmark$ & - & $\checkmark$ & - & $\checkmark$ & $\checkmark$ & $\checkmark$ & - & $\checkmark$ & $\checkmark$ & - & 8 \\
\hline 9 & $\checkmark$ & - & - & - & $\checkmark$ & $\checkmark$ & $\checkmark$ & - & $\checkmark$ & $\checkmark$ & $\checkmark$ & - & $\checkmark$ & $\checkmark$ & $\checkmark$ & 10 \\
\hline 10 & $\checkmark$ & - & - & - & $\checkmark$ & - & - & - & - & $\checkmark$ & - & - & - & - & - & 3 \\
\hline TOTAL & 9 & 2 & 4 & 3 & 8 & 8 & 8 & 3 & 4 & 6 & 9 & 6 & 6 & 5 & 7 & 87 \\
\hline
\end{tabular}

\section{Discusión e implicaciones didácticas}

A pesar de la considerable atención que se ha dado a la problemática de la contaminación por plásticos en los mares por parte de asociaciones ecologistas que a su vez lo han hecho público a través de diversos medios de comunicación, ésta no ha alcanzado el nivel divulgativo que cabría esperar. El desconocimiento que muestran nuestros estudiantes, evidencia el desafío pendiente que existe para involucrar a los jóvenes en la conservación del medio ambiente.

El planteamiento de una situación real como ejemplo de las consecuencias de la contaminación marina, debería haber facilitado que los futuros docentes se aproximaran a la complejidad de estas situaciones, integrando los aspectos sociales, además de los de tipo ambiental. Sin embargo, alcanzar una visión sistémica en la que se incorporen a la escena los aspectos socioeconómicos, se señala como una de las principales dificultades en el proceso de formación de ciudadanos críticos ante la crisis ambiental actual (Van Weelie y Wals 2002). El hecho de que se centren más en supuestos riesgos para la salud y la economía pesquera, nos advierte de la necesidad de poner un mayor énfasis en mostrar la globalización de las consecuencias y facilitar la toma de conciencia sobre la diversidad de pueblos que comparten el planeta y se ven afectados por nuestras decisiones.

En nuestra sociedad, la mayoría de la población percibe las problemáticas ambientales alejadas de su realidad ya que sus efectos son a medio y largo plazo y no afecta a todos por igual (Meira 2013). Los futuros profesores actúan desde esta perspectiva, por mucho que se muestren preocupados ante la situación, analizan y buscan soluciones al problema desde su reducida realidad y obvian el resto del mundo.

Los estudiantes construyen un razonamiento causal simple y concreto sobre la relación existente entre ciertas actividades humanas y las problemáticas ambientales. Sin embargo, su 
comprensión requiere conocer los fenómenos derivados de estas interacciones. Estos conocimientos serán indispensables para analizar desde posiciones críticas las consecuencias de ciertos comportamientos y estilos de vida.

Por otra parte, los futuros docentes parecen haber relacionado la existencia del vórtice con su propio consumo. A este fin, parece determinante el análisis de sus propios residuos. Estas estimaciones podrían haber favorecido una mejor perspectiva sobre el exceso de consumo de determinados productos y la dificultad en la gestión de los residuos. A este efecto, parecen asumir la necesidad de reducir su propio consumo, en especial de plásticos, y proponen fórmulas sencillas que podrían configurar nuevos hábitos más sostenibles.

En cualquier caso, somos conscientes de que reconocer la necesidad de estos cambios no implica que los sujetos los integren en sus vidas, aunque sí es importante que interioricen que son parte del problema y que pueden contribuir a su solución.

A pesar de la disponibilidad que muestran los profesores en formación a asumir compromisos y responsabilidades, para que vayan más allá de meras declaraciones, se necesitaría avanzar más en intervenciones que logren modificar sus percepciones y reconozcan que los hábitos individuales pueden suponer cambios globales. La falta de conciencia sobre cómo nos pueden afectar estos problemas introduce un germen de incertidumbre, ambivalencia y desconcierto en nuestra sociedad desarrollada que puede conducir a un bloqueo de las acciones individuales y colectivas que dificulta la búsqueda de soluciones efectivas (Uzzell 2000).

Después de la intervención educativa, gran parte de los participantes se resisten a asumir responsabilidades individuales sobre el problema, a pesar de los datos de consumo de plástico determinados por ellos mismos. Según Almeida, García y Sánchez (2016) es importante conocer cómo afrontan estas problemáticas ambientales desde su responsabilidad individual, ya que incidirán en sus propuestas de enseñanza-aprendizaje.

En relación a la competencia didáctica que muestran a través del diseño de una salida a la playa, de modo general, ponen especial énfasis en los procesos de discusión y confrontación de ideas que, según ellos, llevarían al alumnado de Primaria a asumir compromisos de tipo individual y colectivo similares a los suyos.

En general, diseñan actividades en las que establecen relaciones causales sencillas y directas entre su propuesta y los posibles compromisos alcanzados por los niños y niñas de Primaria. La mayoría no parten de las ideas de los participantes sobre el problema, sino que comienzan proporcionando la información que consideran relevante, principalmente sobre las consecuencias del problema. Estas cuestiones, según ellos, promueven el interés y se relacionan automáticamente con la recogida de residuos en la playa. Los resultados de esta tarea les servirán para reflexionar sobre sus hábitos y les invitarán a modificarlos.

Los vínculos que pretenden establecer entre la contaminación por plásticos y los hábitos de consumo de los escolares se pueden considerar discretos. En sus propuestas, las tareas están dirigidas en exclusiva a los escolares, las familias tienen un papel secundario, que eventualmente es mencionado. Esta cuestión llama especialmente la atención, ya que en el informe final, una gran parte de los futuros maestros señalaban la importancia de involucrar a las familias para lograr una modificación real de las actividades cotidianas.

Desde el punto de vista de los objetivos de la investigación, el planteamiento de este problema socioambiental ha permitido a los futuros profesores identificar las conexiones entre los impactos en la biodiversidad marina y algunas actividades cotidianas que generan gran cantidad de residuos. Sin embargo, les has resultado difícil explicar el origen del vórtice y sobre todo, relacionarlo con sus hábitos personales de consumo. Por lo tanto, el conocimiento 
de estas conexiones no les ha llevado directamente a asumir responsabilidades y compromisos de cambio. Todo esto revela, sin duda, la dificultad real de pasar de la concienciación a la acción. El proceso que va desde el conocimiento hasta la conducta responsable es bastante complejo y muchos factores de tipo conflictivo y competitivo conforman nuestras acciones y decisiones diarias (Kollmuss y Agyeman 2002).

Respecto a sus propuestas didácticas, tienden a situar la información sobre el problema al inicio de las actividades. Utilizan un modelo simple, según el cual, la visualización de las consecuencias de la contaminación por plásticos será suficiente para motivar a los niños y niñas de Primaria y generar actitudes que derivarán en cambios en sus hábitos de consumo y una mayor concienciación sobre la conservación de los mares y océanos, sin ser consecuentes con las estrategias utilizadas para presentar la información durante la intervención educativa.

En consecuencia, se han puesto de manifiesto algunas dificultades de los futuros profesores al concretar estrategias educativas que permitan plantear los conflictos socioambientales desde una visión sistémica del medio y facilitar el desarrollo del pensamiento crítico en los niños y niñas. Aunque estas circunstancias difícilmente pueden ser superadas por una intervención educativa puntual, una mayor incidencia en este tipo de propuestas podría contribuir a mejorar sus criterios gracias a la selección de actividades que puedan poner en práctica en las aulas de Primaria.

Por último, señalar la importancia de ofrecer estos espacios en la formación inicial de los maestros para incrementar su espíritu crítico respecto a la importancia de nuestro estilo de vida en la generación de los problemas ambientales. Disponer de estas oportunidades, podría aumentar su seguridad al abordar estas temáticas durante su futuro profesional, centrándolas en promover actitudes y comportamientos pro-ambientales, más que en proporcionar información sobre los problemas ambientales.

\section{Referencias}

Almeida A., García B., Sánchez G. (2016). Assessment of pre-service teachers' knowledge of the impact of livestock production on global warming: a comparative study between Portugal and Spain. International Journal of Environmental Studies 73(6), 939-953.

Aramburu, F. (2000). Medio Ambiente y educación. Madrid: Síntesis de Educación.

Blaser M., Feit H.A., McRae G. (2004). In the way of development: Indigenous peoples, life projects and globalization. Ottawa: International Development Research Centre.

Cebrián G., Junyent M. (2014). Competencias profesionales en Educación para la Sostenibilidad: un estudio exploratorio de la visión de los futuros maestros. Enseñanza de las Ciencias 32(1), 29-49.

CESIRE-CDEC (2008). Els criteris de riquesa competencial. https://drive.google.com/file/d/0B_J77cuWFMemNjJIM2M0YTQtNDY5NS00Mm YxLWJiZWQtYWYyZGJiYzljNWE5/view?ddrp=1\&hl=ca\#

Collins-Figueroa M. (2012). Biodiversity and Education for Sustainable Development in Teacher Education Programmes of Four Jamaican Educational Institutions. Journal of Education for Sustainable Development 6(2), 253-267.

Comisión Europea (2018). Our Oceans, Seas and Coast. Descriptor 10: Marine litter. http://ec.europa.eu/environment/marine/good-environmental-status/descriptor-

10/index_en.htm 
Cortina A. (2002). Por una ética del consumo. Madrid: Santillana Ediciones Generales.

Elster D., Barendziak T., Haskamp F., Kastenholz L. (2014). Raising Standards through INQUIRE in Pre-Service Teacher Education. Science Education International 25(1), 29-39.

Eriksen M., Lebreton L.C., Carson H.S., Thiel M., Moore C.J., Borerro J.C., Galgani F., Ryan P. G., Reisser J. (2014). Plastic pollution in the world's oceans: more than 5 trillion plastic pieces weighing over 250,000 tons afloat at sea. PloS One 9(12), e111913.

Esteve, P. (2016). Los futuros maestros ante problemáticas sobre la conservación de la biodiversidad y su utilización en las aulas de Educación Primaria. Tesis doctoral. Universidad de Murcia.

Gallup Organization (2010). Attitudes of Europeans towards the issue of biodiversity Analytical report -Wave 2 - Flash Eurobarometer 290. Bruselas: European Commission.

Gámez L. (2010). Tratamiento del tema transversal Educación para el Consumo en el Alumnado de Tercer Ciclo de Educación Primaria de la Provincia de Granada. Universidad de Granada.

Gayford C. (2000). Biodiversity Education: A Teacher's Perspective. Environmental Education Research 6(4), 347-361.

Grace M., Byrne J. (2010). Engaging pupils in decision-making about biodiversity conservation issues. School Science Review 91(336), 73-80.

Greenpeace (2016). Plásticos en los océanos. Datos, comparativa e impactos. http://www.greenpeace.org/espana/Global/espana/2016/report/plasticos/plasticos_ en_los_oceanos_LR.pdf

Happiness Planet Index (2012). A global index of sustainable well-being. http://www.happyplanetindex.org/

Kollmuss A., Agyeman J. (2002). Mind the Gap: why do people act environmentally and what are the barriers to pro-environmental behaviour? Environmental Education Research 8(3), 239-260.

López Rodríguez R., Jiménez Aleixandre M.P. (2001). Qué tipo de educación ambiental concibe y ejecuta el profesorado. ¿Se hace la misma qué se piensa? ADAXE, Revista de Estudios e Experiencias Educativas 17, 287-309.

Lindemann-Matthies, P. (2002). The influence of an educational program on children's perception of biodiversity. International Journal of Science Education 33(2), 22-31.

Lindemann-Matthies P., Constantinou C., Lehnert H.J., Nagel U., Raper G., Kadji C. (2011). Confidence and Perceived Competence of Preservice Teachers to Implement Biodiversity Education in Primary Schools- Four comparative case studies from Europe. International Journal of Science Education 16(1), 2247-2273.

Meira, P. (2013). Problemas ambientales globales y educación ambiental: Una aproximación desde las representaciones sociales del cambio climático. Revista Integra Educativa 6(3), 29-64.

Ministerio de Agricultura, Pesca, Alimentación y Medio Ambiente (2017) Programa de seguimiento de basuras marinas en playas. Informe de resultados -2017. https://www.mapama.gob.es/es/costas/temas/proteccion-medio-marino/informe 2017 tcm30-455446.pdf

Morgensen F., Mayer M., Breiting S., Varga A. (2009). Educación para el desarrollo sostenible. Tendencias, divergencias y criterios de calidad. Monografías de educación ambiental. 12. Barcelona: GRAO y SCEA. 
Plastic Europe (2017). The Facts-2017. An analysis of European plastics production, demand and waste.https://www.plasticseurope.org/application/files/1715/2111/1527/Plastics_the _facts_2017_FINAL_for_website.pdf

Sims, L., Falkenberg, T. (2013). Developing competencies for education for sustainable development: A case study of Canadian faculties of education. International Journal of Higher Education 2(4), 1-14.

Tilbury D. (2011). Educación para el Desarrollo Sostenible. Examen por los expertos de los procesos y el aprendizaje. París: UNESCO.

UNEP (2009). Marine Litter: A global Challenge. Nairobi: UNEP. www.unep.org/pdf/unep_marine_litter-a_global_challenge.pdf

Uzzell, D.L. (2000) The psycho-spatial dimension of global environmental problems. Journal of environmental psychology 20(4), 307-318.

Van Weelie D., Wals A.E.J. (2002). Making biodiversity meaningful through environmental education. International Journal of Science Education 24(11), 1143-1156.

Vega-Marcote, P., Varela-Losada, M., Álvarez-Suárez, P. (2015). Evaluation of an educational model based on the development of sustainable competencies in basic teacher training in Spain. Sustainability 7(3), 2603-2622.

Vilches A., Gil D. (2013). La ciencia de la sostenibilidad en la formación del profesorado de ciencias. Revista Eureka sobre Enseñanza y Divulgación de las Ciencias 10(4), 749-762.

Wiek, A., Withycombe, L., Redman, C.L. (2011). Key competencies in sustainability: a reference framework for academic program development. Sustainability Science 6(2), 203-218. 
Anexo. Instrumento de recogida de información durante la intervención

\begin{tabular}{|c|c|c|c|}
\hline \multicolumn{3}{|c|}{ HOJA DE TRABAJO } & HOJA DE INFORME FINAL \\
\hline \multicolumn{3}{|c|}{$\begin{array}{l}\text { 1. ¿Conocias antes la existencia de esta Isla? ¿Te parece importante } \\
\text { que todo el mundo la conociera? ¿Por que?? }\end{array}$} & $\begin{array}{l}\text { 1. Haz un listado de las principales consecuencias de la } \\
\text { contaminación por plásticos en el Mar. }\end{array}$ \\
\hline \multicolumn{3}{|c|}{ 2. ¿Cómo explicas el origen de Isla Basura?. } & $\begin{array}{l}\text { 2. Los residuos que tí produces podnan llegar al mar y formar } \\
\text { parte de la Isla Basura? }\end{array}$ \\
\hline \multirow{2}{*}{\multicolumn{3}{|c|}{$\begin{array}{l}\text { 3. A la rista de la información aportada ¿Qué residuos consideras } \\
\text { que son más abundantes en el mar? } \\
\text { 4. De los residuos plásticos ¿cuáles consideras mís peligrosos? } \\
\text { (Ten en cuenta su rida media) }\end{array}$}} & $\begin{array}{l}\text { 3. Conocer Isla Basura iHa cambiado tus ideas sobre la } \\
\text { importancia y necesidad de gestionar adecuadamente los residuos }\end{array}$ \\
\hline & & & $\begin{array}{l}\text { Yla importanca del reciclaje: Explica que has cambiado } \\
\text { 4. ¿Te consideras responsable de esta situación? } \\
\text { Señala la opción con las que estás más de acuerdo }\end{array}$ \\
\hline \multirow{2}{*}{\multicolumn{3}{|c|}{$\begin{array}{l}\text { 5iQué productos de plástico utilizas en tu rida diania? Calcula } \\
\text { cuántos utilizas a la semama y completa la siguiente tabla: }\end{array}$}} & $\begin{array}{l}\text { No, porque yo no tiro basum al mar, es responsabilidad de } \\
\text { los barcos y la gente que deja restos en la plara. }\end{array}$ \\
\hline & & & $\begin{array}{l}\text { Si, me considero responsable como ciudadano y procuro no } \\
\text { utilizar bolsas de plástico }\end{array}$ \\
\hline \multirow[t]{2}{*}{ PRODUCTO } & $\begin{array}{l}\text { N TOTAL EN } \\
\text { LA SEMANA }\end{array}$ & $\begin{array}{l}\text { N TOTAL EN } \\
\text { UNANNO }\end{array}$ & Sólo en parte, porque si esos residuos los recogieran del mar \\
\hline & & & $\begin{array}{l}\text { Sí, el estilo de consumo de nuestra sociedad es el } \\
\text { responsable. }\end{array}$ \\
\hline \multicolumn{3}{|c|}{$\begin{array}{l}\text { 6. Crees que podras reducir el consumo de alguno de estos } \\
\text { productos. En su caso, explica lo que harias. }\end{array}$} & 5. ¿Qué soluciones riables piensas que podria tener este problema? \\
\hline
\end{tabular}

